\section{Ce mois-ci dans Diesen Monat in}

\section{Dernetiology}

\author{
Dermatology 2001;202:87-89
}

\section{Mesure électrique de l'hydratation de la couche cornée: mythes et réalités}

Par ce commentaire critique d'un chapitre de livre [Skin Bioengineering: Techniques and Applications in Dermatology and Cosmetology, Elsner P, et al (eds), Basel, Karger, 1998], les auteurs font une mise au point sur ces techniques de biométrie cutanée. Ils dénoncent les théories obsolètes sur l'impédance cutanée et les fausses informations sur les méthodes de mesure de l'hydratation de la couche cornée qui restent ancrées dans la littérature. Peu d'articles récents sont effectivement consacrés à ce sujet malgré les progrès réalisés ces 10 dernières années. Une discussion technique pour les spécialistes de ce domaine de recherche qui intéresse la dermatologie et la cosmétologie. (I.M.)

\section{Fakten und Mythen der elektrischen Messung der} Hydratation des Stratum corneum

Es handelt sich um einen Kommentar bezüglich des gleichnamigen Kapitels im Buch «Skin Bioengineering: Techniques and Applications in Dermatology and Cosmetology» [Elsner P et al (eds), Basel, Karger, 1998]. Es handelt sich um sehr spezifische technische Daten, mit denen die Autoren darauf aufmerksam machen, dass Hydratationsmessungen des Stratum corneum eigenen Gesetzen folgen und dass diese mit spezifischen Kenntnissen durchgeführt werden müssen. (M.H.)

Facts and Myths about Electrical Measurement of Stratum corneum Hydration State

Orjan G. Martinsen ${ }^{\mathrm{a}}$, Sverre Grimnes $\mathrm{a}, \mathrm{b}$

a Department of Physics, University of Oslo, and

${ }^{b}$ Department of Biomedical and Clinical Engineering, Rikshospitalet, Oslo, Norway

\section{Dermatology 2001;202:90-93}

\section{Expression de Bcl-2 dans le pathergy-test cutané au cours de la maladie de Behçet}

Le pathergy-test cutané est un test clinique aidant au diagnostic de la maladie de Behçet, montrant une hyperréactivité cutanée avec une vasculite leucocytoclasique et lymphocytaire aux points de ponction. Bcl-2 est un proto-oncogène qui retarde la mort par apoptose de la cellule qui l'exprime. Les auteurs montrent que dans 12 cas sur 13 de vasculite sur pathergy-test cutané chez des patients atteints de maladie de Behçet $(n=23)$, les lymphocytes présents dans l'infiltrat expriment Bcl-2, alors que cette expression n'est retrouvée que dans 3 cas sur 11 de vasculite non liée à la maladie de Behçet. L'expression de Bcl2 dans ces lymphocytes réactifs, et donc le retard de leur mort par apoptose, pourrait donc contribuer à la pathogénie de la vasculite dans la maladie de Behçet. (E.L.)

\section{Bcl-2-Expression in der Hautreaktion der Behçet-Krankheit}

Bcl-2 ist ein Schutzfaktor vor Apoptose. Die Autoren haben bei 23 Patienten mit Behçet-Krankheit und 7 Kontrollpersonen einen Pathergietest durchgeführt und $48 \mathrm{~h}$ später Hautbiopsien entnommen. Als Kontrolle diente unbefallene Haut von 7 Patienten mit Behçet-Krankheit und von 11 Patienten mit leukozytoklastischer Vaskulitis. Bei 9 der 23 Patienten war der Pathergietest klinisch positiv. Histologisch war dieser bei 13 Patienten positiv. In der Gruppe der Kontrollpatienten waren beide Tests stets negativ. Bei 12 der 13 positiven Histologien waren die meisten Leukozyten Bcl-2-positiv. Lediglich bei 3 Patienten mit leukozytoklastischer Vaskulitis fanden sich Bcl-2-positive Zellen. (M.H.)

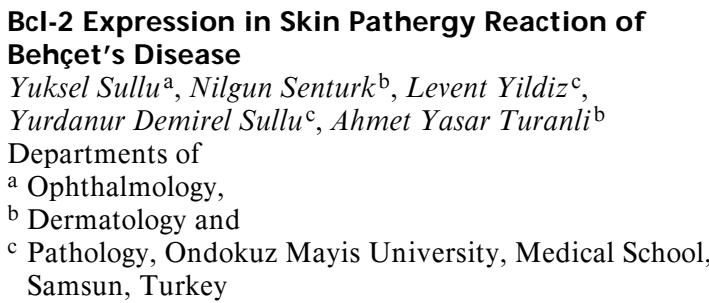

Bcl-2 Expression in Skin Pathergy Reaction of Behçet's Disease

Yuksel Sullu $^{\mathrm{a}}$, Nilgun Senturk ${ }^{\mathrm{b}}$, Levent Yildiz ${ }^{\mathrm{c}}$,

Yurdanur Demirel Sulluc ${ }^{\mathrm{c}}$, Ahmet Yasar Turanli ${ }^{\mathrm{b}}$

Departments of

a Ophthalmology,

b Dermatology and

c Pathology, Ondokuz Mayis University, Medical School, Samsun, Turkey

\section{Dermatology 2001;202:94-98}

Rôle du granzyme B lymphocytaire dans l'atteinte épidermique de la maladie de réaction du greffon contre l'hôte

La réaction cutanée du greffon contre l'hôte $(\mathrm{GVH})$ est caractérisée par un infiltrat lymphocytaire et une apoptose des kératinocytes épidermiques. Cette mort des kératinocytes est induite par les lymphocytes T cytotoxiques, et trois grands mécanismes semblent impliqués: l'exocytose de granules cytotoxiques dont la perforine et les granzymes sont des constituants majeurs; l'interaction Fas/Fas ligand; des médiateurs solubles tels que le TNF- $\alpha$. Des immunomarquages pour le granzyme B ont été effectués sur les biopsies cutanées de 8 patients qui avaient développé une GVH aiguë et/ou chronique après une greffe de moelle. Le granzyme $\mathrm{B}$ a été mis en évidence dans les lymphocytes $\mathrm{T}$ cytotoxiques (CD8+) intra-épidermiques de $3 / 6$ GVH aiguës et 5/6 GVH chroniques (phase lichénoïde). Dans la GVH chronique, il était également retrouvé libre dans les espaces intercellulaires autour des kératinocytes basaux. Ces résultats suggèrent un rôle du granzyme B lymphocytaire dans le mécanisme lésionnel épidermique de la GVH, en particulier dans la phase chronique lichénoïde. (I.M.)

\section{Beteiligung von Granzyme-B-haltigen} Lymphozyten bei epidermalen Läsionen in der Transplantat-Wirt-Reaktion

Granzyme B und Perforin sind bei der durch zytotoxische T-Zellen induzierten Apoptose beteiligt. Die Autoren stellten sich die Frage, ob diese beiden Substanzen für die Apoptose in Hautläsionen im Rahmen einer Transplantat-Wirt-Reaktion (GVHD) verantwortlich zu machen sind. Sie unternahmen eine immunhistochemische Studie bei 8 Patienten mit GVHD nach Knochenmarktransplantation. Granzyme-B-positive Lymphozyten waren CD8-positiv und konnten in 3 von 6 Pro- 
ben von akuter GVHD und in 5 von 6 Proben von chronischer GVHD gefunden werden. Perforin-positive Lymphozyten wurden lediglich jeweils bei einer Probe von chronischer und akuter GVHD gefunden (M.H.)

\section{Granzyme-B-Containing Lymphocyte Involvement in} Epidermal Injury in Graft-versus-Host Disease

Yoko Higaki a , Osamu Yamadab, Takamitsu Okamurab,

Hideaki Mizoguchi ${ }^{\mathrm{b}}$, Makoto Kawashima ${ }^{\mathrm{a}}$

Departments of

a Dermatology and

b Haematology, Tokyo Women's Medical University, Tokyo, Japan

\section{Dermatology 2001;202:99-107}

\section{Aspects cliniques et immunologiques du syndrome de Comèl-Netherton}

Ce syndrome rare associe une ichtyose, des anomalies pilaires, une diathèse atopique (exzema résistant au traitement, asthme, urticaire, allergies alimentaires, IgE très élevées) et un déficit immunitaire. Les 5 observations décrites soulignent la diversité des signes cliniques et le pronostic très variable du syndrome de Comèl-Netherton ( 2 enfants sont décédés dans la premiere année). Une analyse des cytokines impliquées dans la régulation de la synthèse des IgE (IL-4, -5, -13, IFN- $\gamma$ ) a été effectuée chez 2 patients et chez des enfants normaux et atopiques (expression du ARNm et production de la protéine par les cellules mononucléées du sang circulant et les lymphocytes $\mathrm{T}$ en culture). Dans 1 cas, il y a une faible production de cytokines de type Th1 (IL-4, -5, -13) et Th2 (IFN- $\gamma$ ); dans l'autre, il y a une activation préférentielle des cellules produisant les cytokines Th2. Le pourcentage de cellules «natural killer» (productrices d'IFN- $\gamma$ ) est nettement abaissé chez les 2 patients. Cet article apporte quelques données sur les anomalies immunitaires du syndrome de Comèl-Netherton et montre que les mécanismes responsables de l'hyper-IgE sont variables et complexes. (I.M.)

\section{Klinisch-immunologische Heterogenie beim Comèl-Netherton-Syndrom}

Es werden 5 Kinder mit Comèl-Netherton-Syndrom vorgestellt. Bei 2 davon wurde eine Analyse der Zytokine, die die IgE-Synthese regulieren, durchgeführt. Es zeigte sich, dass bei diesen Patienten ein ganz auffälliger Unterschied bezüglich der Schwere der Erkrankung und des Krankheitsverlaufs bestand. Auffällige Unterschiede konnten durch Analyse der verschiedenen Zytokine demonstriert werden. Die Anzahl der «Natural-Killer»-Zellen war bei 2 untersuchten Patienten sehr niedrig. (M.H.)

\section{Clinico-Immunological Heterogeneity in}

Comèl-Netherton Syndrome

D. Van Gysel ${ }^{\mathrm{a}, \mathrm{d}}$, H. Koning ${ }^{\mathrm{b}, \mathrm{c}}$, M.R.M. Baert ${ }^{\mathrm{c}}$

H.F.J. Savelkoul ${ }^{\mathrm{c}}$, H.J. Neijens ${ }^{\mathrm{b}}$, A.P. Oranje ${ }^{\mathrm{d}}$

a Department of Paediatrics, O.L. Vrouw Hospital, Aalst,

Belgium;

b Department of Paediatrics, University Hospital (Sophia)

Rotterdam,

c Department of Immunology, Erasmus University Rotterdam, and

d Department of Dermatology and Venereology, University Hospital Rotterdam, and Erasmus University Rotterdam, The Netherlands

\section{Dermatology 2001;202:108-115}

\section{Photothérapie du psoriasis:}

expérience comparative de différentes approches photothérapeutiques

Dans cette étude rétrospective monocentrique allemande, les auteurs analysent 203 patients traités pour un psoriasis en plaque sévère (PASI de 12-35) par photothérapie, et comparent les trois protocoles utilisés: PUVA (19 patients), balnéoPUVA (87 patients), photothérapie UVB (97 patients). Les trois protocoles étaient comparables en terme d'efficacité (rémission complète dans $70-90 \%$ des cas), mais les auteurs semblent préférer la balnéo-PUVA comme ayant moins d'effets secondaires (15 vs. $30 \%$ pour les autres, avec cependant trois réactions bulleuses en balnéo-PUVA) et une durée de rémission plus longue (8.4 vs. 5 mois pour la photothérapie UVB). (E.L.)

\section{Phototherapie der Psoriasis: \\ Vergleichende Untersuchungen der verschiedenen phototherapeutischen Möglichkeiten}

Es handelt sich um eine retrospektive Studie, in der $203 \mathrm{~Pa}-$ tienten mit verschiedenen Stadien von Psoriasis einbezogen wurden. Verglichen wurden UVB mit und ohne Anthralin oder Calcipotriol mit der Bade-PUVA-Therapie. Hierbei wurden Wirksamkeit, Behandlungsdauer, Behandlungsfrequenz, kumulative Lichtdosis, Nebenwirkungen sowie Remissionsdauer untersucht. Betreffend der Wirksamkeit bestand kein signifikanter Unterschied zwischen Bade-PUVA-Therapie und UVBPhototherapie. Die Behandlungsdauer war bei Bade-PUVATherapie deutlich länger, jedoch wies diese Therapieform im Vergleich weniger Nebenwirkungen auf. Bezüglich der Remissionsdauer bestanden ebenfalls Vorteile seitens der BadePUVA-Therapie. (M.H.)
Phototherapy of Psoriasis: Comparative Experience of Different Phototherapeutic Approaches
Sigrid Karrer, Christian Eholzer, Günther Ackermann, Michael Landthaler, Rolf-Markus Szeimies
Department of Dermatology, University of Regensburg, Germany 
Le tacalcitol topique n'améliore pas les ichthyoses par rétention

Cet analogue synthétique de la vitamine $\mathrm{D}_{3}$ (1 $\alpha, 24$-dihydroxyvitamine $\mathrm{D}_{3}$ ) a été testé dans 5 cas d'ichthyose liée à l'X, 3 d'ichthyose vulgaire et 1 ichthyose acquise. Les mécanismes physio-pathogéniques de ces ichthyoses sont très différents, mais elles sont toutes caractérisées par une hyperkératose de type rétentionnel, sans hyperprolifération épidermique. Le tacalcitol $(2 \mu \mathrm{g} / \mathrm{g})$ a été appliqué chaque jour sur la jambe droite et le véhicule (vaseline) sur la jambe gauche. Après 8 semaines, il y avait une amélioration symétrique de l'état cutané; le tacalcitol n'a donc apporté aucun bénéfice par rapport à l'excipient. Cette étude porte sur un petit groupe hétérogène de patients, mais elle confirme ce qui avait déjà été constaté avec la $1 \alpha$-hydroxyvitamine $\mathrm{D}_{3}$ et le calcitriol, c'est-à-dire l'absence d'effet thérapeutique des dérivés de la vitamine $\mathrm{D}_{3}$ dans ce type d'ichthyoses sans hyperprolifération épidermique. (I.M.)

\section{Bestimmung der klinischen Wirksamkeit von topischem Tacalcitol bei Ichthyose mit retentioneller Hyperkeratose}

Mehrere Patienten mit verschiedenen Formen von retentioneller Hyperkeratose wurden mit Tacalcitol in einem Rechts-Links-Vergleich mit der Salbengrundlage untersucht. Hierbei zeigte sich, dass das topische Tacalcitol nicht wirksamer als das Vehikel alleine war. (M.H.)

Assessment of the Clinical Effect of Topical Tacalcitol on Ichthyoses with Retentive Hyperkeratosis

M. Okano

Department of Dermatology, Aizembashi Hospital, Osaka, Japan

\section{Traitement de la maladie de Kaposi classique (méditerranéenne) par gemcitabine}

Les auteurs ont traité 11 patients atteints de maladie de Kaposi classique résistante aux chimiothérapies classiques par gemcitabine, un nouvel agent antitumoral actif sur les cancers solides et ayant peu d'effets secondaires. Une réponse complète et 10 réponses partielles ont été observées, avec très peu d'effets secondaires ( 2 cas de lymphopénie et d'élévation des transaminases modérées). La gemcitabine pourrait constituer à l'avenir une arme thérapeutique de choix dans la maladie de Kaposi classique. (E.L.)

\section{Behandlung von klassischem Kaposi-Sarkom mit Gemcitabin}

Gemcitabin ist ein Analog des Deoxycytidins, das eine zytotoxische Wirkung auf solide Tumoren und gleichzeitig wenig Nebenwirkungen hat. 12 Patienten mit der aggressiven rekurrenten Form des Kaposi-Sarkoms wurden mit diesem Medikament behandelt. Nach Ausschluss eines Patienten konnten bei 1 von 11 Patienten eine komplette Remission und bei 10 der 11 Patienten eine partielle Remission erzielt werden. Die Toxizität war hierbei eingeschränkt. Somit stellt diese Substanz bei dieser Indikation, insbesondere bei therapieresistenten Fällen, einen ganz erheblichen Vorteil dar. (M.H.)

\footnotetext{
Treatment of Classical Kaposi's Sarcoma with

Gemcitabine

Lucia Brambilla $^{\text {a }}$, Roberto Labianca ${ }^{\text {b }}$,Silvia M. Ferrucci ${ }^{\text {a }}$,

Michela Taglioni ${ }^{\text {a }}$, Vinicio Bonesch ${ }^{\text {a }}$

${ }^{a}$ Institute of Dermatology, State University, IRCCS Ospedale

Maggiore Policlinico, Milan, and

${ }^{\mathrm{b}}$ Unit of Medical Oncology, Ospedali Riuniti, Bergamo, Italy
}

\section{Avec les compliments des éditions S. Karger, Bâle}

Mit den besten Empfehlungen des Verlags S. Karger, Basel 